\title{
Evaluation of 5G Modulation Candidates WCP-COQAM, GFDM-OQAM, and FBMC-OQAM in Low-Band Highly Dispersive Wireless Channels
}

\author{
Aitor Lizeaga, ${ }^{1}$ Pedro M. Rodríguez, ${ }^{2}$ Iñaki Val, ${ }^{2}$ and Mikel Mendicute ${ }^{1}$ \\ ${ }^{1}$ Signal Theory and Communications Area, Mondragon University, Mondragón, Spain \\ ${ }^{2}$ Department of Communications, IK4-IKERLAN, Mondragón, Spain \\ Correspondence should be addressed to Aitor Lizeaga; alizeaga@mondragon.edu
}

Received 16 December 2016; Revised 17 March 2017; Accepted 15 August 2017; Published 16 October 2017

Academic Editor: Lei Zhang

Copyright (c) 2017 Aitor Lizeaga et al. This is an open access article distributed under the Creative Commons Attribution License, which permits unrestricted use, distribution, and reproduction in any medium, provided the original work is properly cited.

\begin{abstract}
We analyse some of the candidates for modulations for 5G: FBMC-OQAM, GFDM-OQAM, and WCP-COQAM. Unlike most of the related bibliographies, which are oriented to mobile communications, our research is focused on 5G in cognitive radio based industrial wireless communications. According to the ultrareliability and low-latency requirements of industrial communications, we simulate the aforementioned modulations in low-band transmissions (carrier frequencies below $6 \mathrm{GHz}$ and a bandwidth narrower than $100 \mathrm{MHz}$ ) through large indoor spaces and severe multipath channels that emulate industrial halls. Moreover, we give detailed information about WCP-COQAM and how the windowing affects the protection against multipath effect and reduces spectral efficiency compared to GFDM-OQAM. We also compare the aforementioned filtered multicarrier techniques and OFDM in terms of robustness against multipath channels, power spectral density, and spectral efficiency. Based on these results, we aim at providing an approximate idea about the suitability of 5G MCM candidates for industrial wireless communications based on CR.
\end{abstract}

\section{Introduction}

Multicarrier modulation (MCM) schemes are widely adopted in wireless communication systems, orthogonal frequency division multiplexing (OFDM) being the most extended one among them. Despite the features and benefits that OFDM offers in wireless communications, it also presents significant drawbacks such as high out-of-band (OOB) radiation. Considering this fact and the upcoming applications and needs in wireless communications, a lot of research on new waveforms for the physical (PHY) communication layer of the fifth generation $(5 \mathrm{G})$ has been carried out in order to overcome OFDM's limitations [1-4]. As a result, several pulseshaping techniques exist, which allow subcarrier filtering in MCM systems. These techniques provide good frequency localization and reduce the OOB radiation. Among the main PHY candidates for 5G, the most extended MCM schemes in the bibliography are filter-bank multicarrier offset quadrature amplitude modulation (FBMC-OQAM) [5], generalized frequency division multiplexing (GFDM) [6], and universal filtered multicarrier (UFMC) [7]. FBMC-OQAM and GFDM consist in filtering each subcarrier in order to get good frequency localization in each subcarrier; UFMC, on the contrary, consists in filtering groups of subcarriers in order to reduce only the OOB radiation.

Although most of the research carried out on PHY proposals for $5 \mathrm{G}$ is oriented to mobile communications, $5 \mathrm{G}$ is actually meant to cover a wide range of application fields [8]. In order to cope with the diversity of services and the flexibility that $5 \mathrm{G}$ will demand, in $[9,10]$ innovative frameworks allowing multiservice subband filtered multicarrier systems are introduced. These frameworks present a structure in which the available spectrum is divided into subbands dedicated to different services. The division and interference avoidance between different services is provided by means of subband filtering.

Among all the application fields that 5G will cover, our research focuses on wireless industrial communications [11]. Specifically, we investigate robust PHY layers designed for industrial wireless communications based on cognitive 
radio (CR) $[12,13]$. Since the existing industrial wireless communication systems based on the IEEE 802.15.4 standard lack robustness against these harsh channel conditions [14, 15], new schemes for the PHY layer in industrial wireless communications must be proposed. This kind of industrial scenarios is characterized by the presence of high impulsive noise and interference from other wireless systems and fading channels due to severe multipath effect and shadowing caused by the numerous, large, and metallic obstacles within the propagation channel. In this work, we simulate transmissions through large indoor spaces and severe multipath channels in order to model some of these characteristics.

Besides the aforementioned harsh environments, requirements for industrial communications suppose an extra challenge for wireless systems, especially in some factory automation (FA) applications. Since $5 \mathrm{G}$ is also meant to cover these industrial communications' ultrareliability and low-latency requirements (up to $10^{-9}$ packet error rates, near $100 \mu$ s overthe-air transmission time, and $\leq 1 \mathrm{~ms}$ end-to-end latency) [1618 ], in this work, we simulate and evaluate some of the MCM $5 \mathrm{G}$ candidates in low-band transmissions (carrier frequencies below $6 \mathrm{GHz}$ and a bandwidth narrower than $100 \mathrm{MHz}$ ).

In this article, among the aforementioned industrial environment characteristics, we focus on severe multipath effect. We analyse FBMC-OQAM, GFDM-OQAM, and also windowed cyclic prefix-circular OQAM (WCP-COQAM) modulation systems. Although WCP-COQAM [19-21] is not as extended as the other two schemes in the bibliography, it shares several similarities with GFDM, which make it another candidate for 5G [22] and an interesting MCM scheme to be analysed. We evaluate their performance in terms of bit error rate (BER), power spectral density (PSD), and spectral efficiency (SE) under highly dispersive channels. We compare these results with traditional OFDM and we use the SE to represent the cost of using cyclic prefix (CP) extensions, subcarrier filtering, and windowing schemes in each MCM system.

Based on those analyses and results, we aim at providing a notion about the suitability of $5 \mathrm{G}$ MCM candidates for industrial wireless communications based on CR.

From the analysis and the assessment of the aforementioned MCM systems, we provide the following contributions:

(1) This article complements the work in [19-21] by bringing additional details about how windowing affects the protection against multipath channels and the SE in WCP-COQAM. We state the conditions to provide full orthogonality in multipath channels and we show how windowing reduces SE in WCPCOQAM compared to GFDM-OQAM. We explain all these details in Sections 2.3, 2.4, and 3 and provide some results and discussion in Section 4.3.

(2) While most research about $5 \mathrm{G}$ is focused on mobile communications, here we simulate and assess some of the main $5 \mathrm{G}$ modulation candidates under different conditions. We simulate low-band transmissions through large indoor spaces and severe multipath channels in order to model some of the wireless

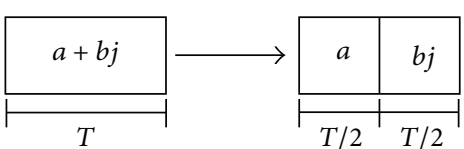

FIGURE 1: Complex to pure real and imaginary operation.

industrial environments' characteristics. Under these conditions and by means of BER, PSD, and SE analysis, we evaluate the suitability of the aforementioned MCM systems for wireless industrial communications based on CR.

The paper is organised as follows: in Section 2, we explain the theoretical background of the MCM systems we are going to analyse; in Section 3, we give more detailed explanations about WCP-COQAM and make some clarifications with respect to the bibliography; in Section 4, we present the results obtained from our simulations, based on which we compare and discuss the performances of the considered MCM systems; in Section 5, we state the conclusions we obtained from our research; finally, in Section 6, we explain which will be the next steps in our investigation.

\section{Multicarrier Modulations}

The MCM systems we analyse are based on OQAM instead of classical IQ modulations (e.g., N-PSK and N-QAM). The reason why we focus on OQAM-based schemes is that MCM systems cannot simultaneously keep good time-frequency localization (TFL), Nyquist symbol rate, and orthogonality between transmitted symbols if conventional IQ modulations are used, as it is stated by the Balian-Low Theorem (BLT) [23]. According to BLT, intersymbol interference (ISI) and intercarrier interference (ICI) introduced by the prototype filters of these MCM systems make it impossible to achieve perfect reconstruction of complex valued symbols transmitted at Nyquist rate. Consequently, in case matched filter receivers are used, FBMC and GFDM perform worse than OFDM in terms of BER. One solution to overcome the constraints defined by the BLT is to send alternately real and imaginary valued symbols at twice the Nyquist rate instead of complex symbols. This technique is known as OQAM [5]. This strategy allows the filtered data symbols to remain orthogonal and keep both good TFL and Nyquist symbol rate, all at the same time.

Hence, we first introduce the OQAM technique and next we explain the rest of the MCM schemes.

2.1. OQAM. OQAM consists, basically, in splitting one complex symbol into two semisymbols, one real and one imaginary. After this operation, the whole OQAM symbol's duration remains equal to the duration of the original complex symbol, while the duration of each semisymbol is half the whole symbol, as shown in Figure 1. Thus, OQAM symbol structure's sampling rate is twice the original complex symbol structure's. It is worth mentioning that this oversampling does not carry any change in the total bandwidth of the 


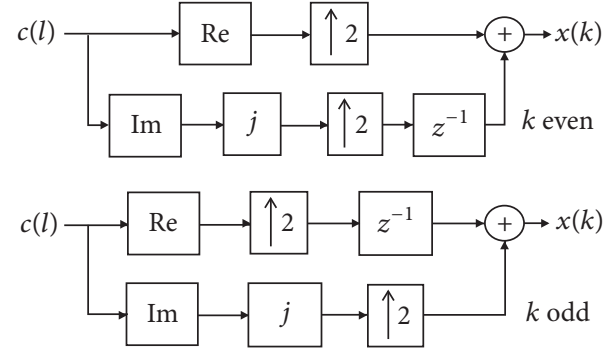

FIGURE 2: Block diagram of the OQAM symbols arrangement in a multicarrier system. For frequency domain alternation, one sample delay is applied to imaginary semisymbols in even subcarriers and to real semisymbols in odd subcarriers.

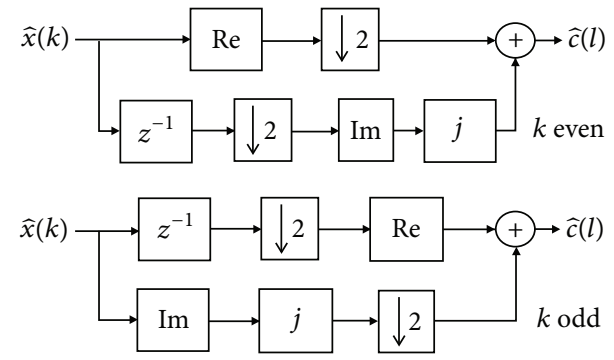

FIGURE 3: Block diagram of the OQAM demodulation process in a multicarrier system. This diagram performs the inverse operation to the one shown in Figure 2.

transmitted signal when OQAM is used in MCM systems. As we explain in Section 2.2, when the multicarrier OQAM signal is serialized, its symbol rate is the same as it would be in a conventional IQ modulation.

The OQAM scheme discussed up to now can reduce ISI caused by pulse-shaping processes. Since imaginary and real semisymbols are transmitted alternately, interference coming from adjacent semisymbols can be ignored in the receiver (i.e., interference caused by a real semisymbol to its adjacent imaginary semisymbol can be ignored and vice versa).

Similarly, in frequency domain, pulse-shaping makes adjacent subcarriers overlap each other, thus causing severe ICI. If those subcarriers are organised so that real and imaginary ones are alternated one next to the other, ICI from adjacent subcarriers will be avoided because interference coming from them can be ignored in the receiver. Figure 2 shows the block diagram of an OQAM MCM system. Figure 3, on the other hand, shows the demodulation process to recover the original complex data symbols.

More details about OQAM can be found in [5].

2.2. FBMC-OQAM. FBMC consists in filtering each subchannel in order to get well-localized subcarriers. Figure 4 shows the basic representation of a FBMC-OQAM transmultiplexer. The main blocks are the OQAM preprocessing or modulator block, the synthesis filter bank, the analysis filter bank, and OQAM postprocessing or demodulator.

The key components of the FBMC-OQAM scheme are the synthesis and analysis filter banks, which are formed by one filter per subchannel. The filter of the $m$ th subchannel is defined as

$$
g_{m}(n)=p(n) \exp \left(j \frac{2 \pi m n}{M}\right),
$$

where $p(n)$ is the prototype filter of length $L_{p}$ and $n=$ $0, \ldots, L_{p}-1$. The exponential factor corresponds to the $m$ th subcarrier, where $M$ is the total number of subcarriers.

Note that the FBMC-OQAM scheme works as a multirate system. Before filtering the $x_{m}(k)$ OQAM data symbols, they are upsampled by $M / 2$ using a zero padding process to get the oversampled signal $a_{m}(n)$ :

$$
a_{m}(n)=\sum_{k \in \mathbb{Z}} x_{m}(k) \delta\left(n-\frac{k M}{2}\right), \quad n \in \mathbb{Z} .
$$

The reason why the upsampling factor is $M / 2$ and not $M$ is that OQAM preprocessing already introduces an upsampling factor of 2. Thus, the FBMC-OQAM signal can be expressed as

$$
y(n)=\sum_{m=0}^{M-1} \sum_{l \in \mathbb{Z}} a_{m}(l) g_{m}(n-l), \quad n \in \mathbb{Z} .
$$

At the receiver side, the FBMC-OQAM signal $y(n)$ is demodulated so that, in first place, each subchannel is filtered in order to get only its corresponding signal:

$$
\widehat{a}_{m}(n)=\sum_{l \in \mathbb{Z}} y(l) g_{m}(n-l), \quad n \in \mathbb{Z},
$$

where $m=0, \ldots, M-1$

Then, $\widehat{a}_{m}(n)$ is sampled in the time domain, so that the estimation of the original OQAM signal $x_{m}(k)$ is obtained:

$$
\widehat{x}_{m}(k)=\widehat{a}_{m}\left(\frac{k M}{2}\right), \quad k \in \mathbb{Z} .
$$

Transmission channel, noise, equalization, and other issues have been omitted in order to focus just on FBMCOQAM waveform modulation and demodulation. More details about FBMC-OQAM can be found in [5].

2.3. GFDM-OQAM. The structure of a GFDM-OQAM transmultiplexer is similar to that of the FBMC-OQAM transmultiplexer shown in Figure 4. GFDM-OQAM is also based on OQAM modulation and subchannel filtering. The difference is that in GFDM-OQAM filtering is performed by a circular convolution instead of the linear convolution used in FBMC-OQAM. This way, the modulation system now adopts a block-based signal structure, so that a CP can be added to provide orthogonality against multipath channels without affecting signal's TFL.

We consider a GFDM-OQAM system with $M$ subchannels and $K$ complex valued data symbols in each block (i.e., $2 K$ OQAM symbols in each block). Thus, if we consider $y(n)$ as one GFDM-OQAM block, it can be expressed as

$$
y(n)=\sum_{m=0}^{M-1} \sum_{l=0}^{M K-1} a_{m}(l) \widetilde{g}_{m}(n-l+M K-1),
$$




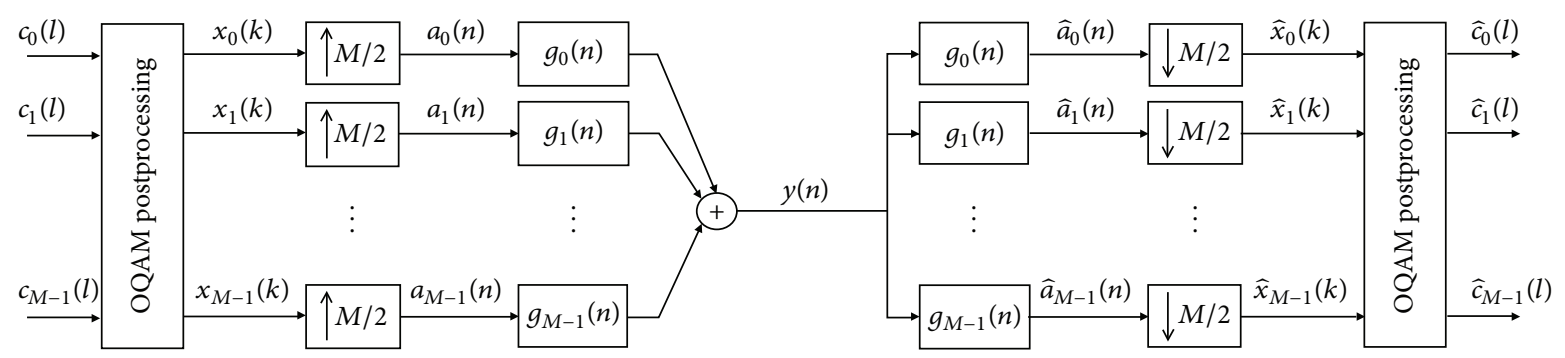

FIGURE 4: Basic representation of a FBMC-OQAM transmultiplexer. This scheme shows the basic idea behind FBMC-OQAM, where each symbol of each subchannel is modulated as OQAM, multiplied by its corresponding subcarrier, and filtered by the prototype filter. In the receiver, the inverse process is performed.

where $n=0, \ldots, M K-1$ and $\widetilde{g}_{m}$ filters are obtained by the periodic repetition of $g_{m}$ filters defined in (1). So $\tilde{g}_{m}(k)=$ $g_{m}[\bmod (k, M K)]$.

At the receiver side, the GFDM-OQAM signal $y(n)$ is demodulated so that, in first place, each subchannel is filtered in order to get only its corresponding signal. Once again, the difference between FBMC-OQAM and GFDM-OQAM receivers consists in the circular convolution:

$$
\widehat{a}_{m}(n)=\sum_{l=0}^{M K-1} y(l) \widetilde{g}_{m}(n-l+M K-1),
$$

where $m=0, \ldots, M-1$.

Then, $\widehat{a}_{m}(n)$ is sampled in the time domain, so that the estimation of the original OQAM signal $x_{m}(k)$ is obtained, just as it is shown in (5).

The insertion of a CP, transmission channel, noise, equalization, and other issues have been omitted in order to focus just on GFDM-OQAM waveform modulation and demodulation. More details about GFDM-OQAM can be found in $[19,20]$. In those works, the idea of GFDM-OQAM is introduced, although the authors refer to this scheme as FBMC-COQAM or just as COQAM.

2.4. WCP-COQAM. WCP-COQAM is introduced in [21]. This technique can be considered as a GFDM-OQAM system with a windowing process, which improves the PSD with respect to GFDM-OQAM.

In WCP-COQAM, both the CP and the windowing are related. The CP is divided into two parts: the guard interval (GI) and the window interval (WI) (referred to as RI in [21]). So the total length of the CP will be equal to the sum of the lengths of the GI and WI parts:

$$
L_{\mathrm{CP}}=L_{\mathrm{GI}}+L_{\mathrm{WI}} \text {, }
$$

where GI is the part of the CP aimed at protecting against multipath channels and WI is the part that will be used for windowing.

Let us consider a GFDM-OQAM signal $s_{\text {GFDM-OQAM }}$ as a queue of several GFDM-OQAM blocks, each with its CP extension of length $L_{\mathrm{CP}}$. Equally, we will consider $M$ to be the total number of subcarriers and $K$ to be the number of symbols in each block. Then the $l$ th block of a WCP-COQAM signal is defined as

$$
\begin{aligned}
& s_{\text {WCP-COQAM }}(k) \\
& =\sum_{r=l-1}^{l+1} s_{\text {WCP-COQAM }}\left(k+r L_{\mathrm{WI}}\right) w(k-r Q),
\end{aligned}
$$

where $k=0, \ldots, M K+L_{\mathrm{CP}}-1 ; \mathrm{Q}=M K+L_{\mathrm{GI}}$, and the window function $w(k)$ is defined by

$$
w(k)
$$

$$
= \begin{cases}\text { window coeff., } & k=0, \ldots, L_{\mathrm{WI}}-1, \\ 1, & k=L_{\mathrm{WI}}, \ldots, M K+L_{\mathrm{GI}}-1, \\ \text { window coeff., } & k=\mathrm{Q}, \ldots, M K+L_{\mathrm{CP}}-1, \\ 0, & \text { otherwise. }\end{cases}
$$

In our work, we use a Hamming window for the window coefficients. Figure 5 shows the block diagram that represents this whole process.

It must be noted from (9) and (10) that the samples of the CP corresponding to the WI part and also the last $L_{\mathrm{WI}}$ samples of the block are multiplied by the window coefficients and they are overlapped with the WI regions of the previous and next blocks, respectively. By overlapping the WI regions, these extra samples are prevented from reducing SE, as shown in Figure 6.

The receiver structure is depicted in Figure 7. It is worth noticing that Figure 7 only shows the recovery process of one GFDM-OQAM block from a received WCP-COQAM signal. Once the GFDM-OQAM signal is recovered, there are several algorithms for its demodulation. In Section 2.3 of this paper, the basic demodulation scheme for GFDM-OQAM is explained and in [21] the authors introduce a computationally efficient demodulation scheme.

In [21], for this scheme, perfect synchronization and an ideal channel are assumed. So, in the first place, the first $M K+L_{\mathrm{GI}}$ samples of one WCP-COQAM block are taken. Next, the first $L_{\mathrm{GI}}$ (which correspond to the CP extension) samples are removed, while the last $L_{\mathrm{WI}}$ samples are left to process them within the next block. Finally, in order to get all the samples back to their original positions within the 


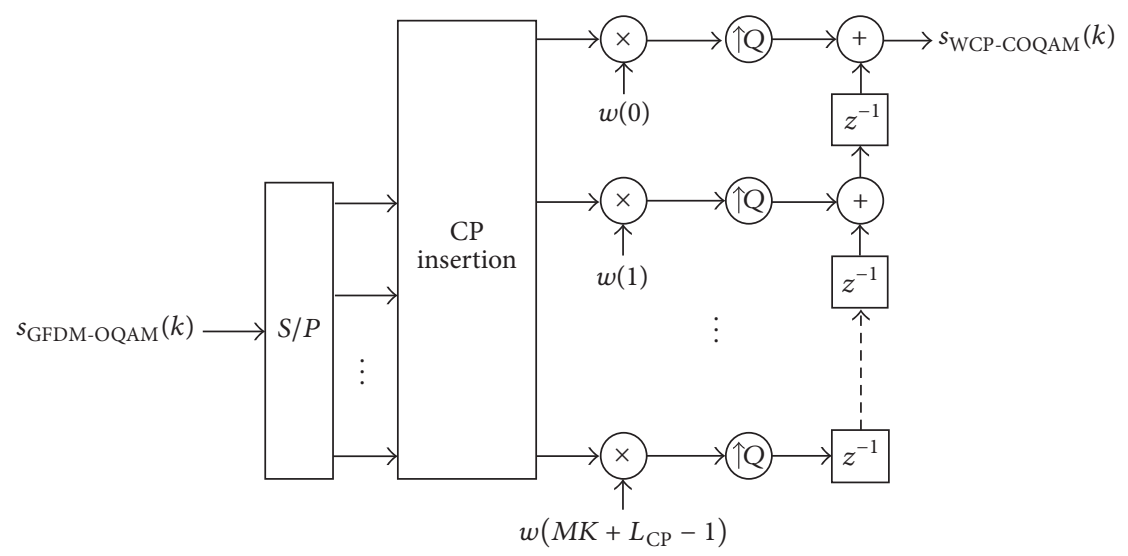

FIGURE 5: Block diagram of the cyclic prefix insertion and windowing operation for WCP-COQAM. A WCP-COQAM signal is made from a GFDM-OQAM signal and a CP and a window function are applied.

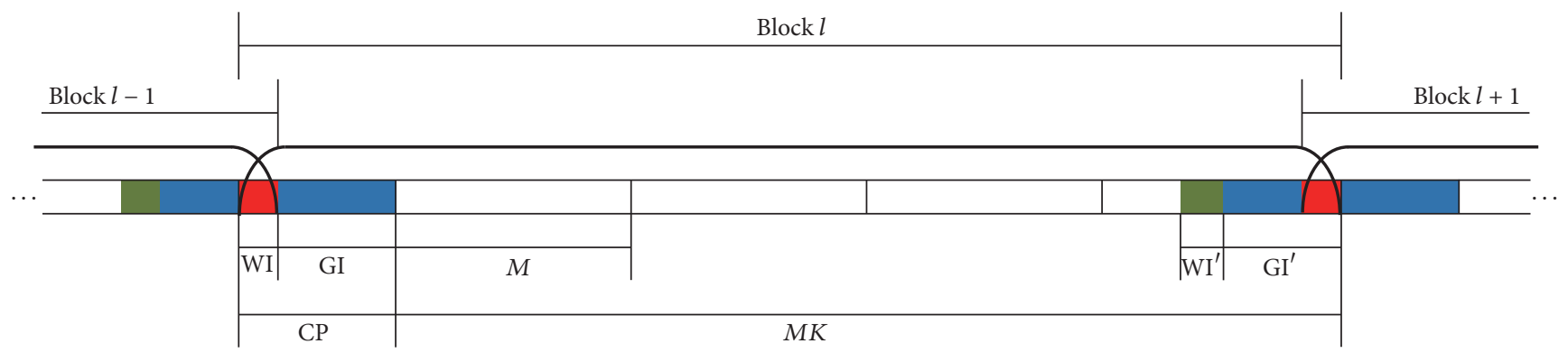

FIGURE 6: Overlapping of adjacent WCP-COQAM blocks. The regions affected by the window of one block are overlapped with the regions affected by the window of the previous and the next blocks. These regions correspond to the first and the last $L_{\mathrm{WI}}$ samples of each block.

original GFDM-OQAM block, a cyclic shift is carried out, which can be expressed as

$$
s_{\mathrm{GFDM}-\mathrm{OQAM}}^{\prime}(k)=s\left[\bmod \left(k+L_{\mathrm{WI}}, M K\right)\right],
$$

where $k=0, \ldots, M K-1 ; s_{\text {GFDM-OQAM }}^{\prime}(k)$ is the estimation of the original $s_{\text {WCP-COQAM }}(k)$ signal, and $s(k)$ is the signal after the removal of the windowing effect and the $\mathrm{CP}$.

\section{Orthogonality Condition and Spectral Efficiency Analysis of WCP-COQAM}

Several analyses on $5 \mathrm{G}$ modulation candidates are available in the bibliography $[24,25]$, some of them even including WCPCOQAM modulation scheme [26, 27]. However, to the best of our knowledge, none of them address the implications of windowing in the WCP-COQAM waveform as we do here.

In this section, we contribute some additional details regarding how windowing affects the orthogonality against multipath channels and the SE in WCP-COQAM. Specifically, we state the condition that the GI region of the $\mathrm{CP}$ extension must fulfil in order to provide full orthogonality in the presence of multipath channels. Besides, we also compare WCP-COQAM and GFDM-OQAM (due to the similarities between both MCM systems) in terms of SE and we show that, in this aspect, the former is outperformed by the latter if equal multipath protection is assumed.
3.1. Orthogonality Condition. In WCP-COQAM, multipath channel protection is directly related to the CP extension and also to the windowing process. In order to explain this fact, we show in detail the steps to form a WCP-COQAM block starting from a basic GFDM-OQAM block (as seen in Section 2.3).

Once a GFDM-OQAM block is formed, the CP extension is added to it. Figure 8(a) shows the details of this first stage in the construction of WCP-COQAM signal, where $M$ is the total number of subcarriers; $K$ is the number of symbols per block; WI is the region of length $L_{\mathrm{WI}}$ devoted to windowing and overlapping; GI is the region of length $L_{\mathrm{GI}}$ that protects against multipath channel effect; and the $\mathrm{CP}$ extension has a total length of $L_{\mathrm{CP}}=L_{\mathrm{WI}}+L_{\mathrm{GI}} \cdot \mathrm{CP}^{\prime}, \mathrm{WI}^{\prime}$, and $\mathrm{GI}^{\prime}$ are those last samples of the block which are copied in order to form the CP extension. As is represented in Figure 8(a), before the windowing is applied, this signal can be considered as a GFDM-OQAM block with CP extension.

Figure 8(b) represents the WCP-COQAM block right after windowing is applied. As shown in Figure 6 and in (9) and (10), the window function is applied on the first and the last $L_{\mathrm{WI}}$ samples, so that these samples are somehow distorted. Because of that, CP, WI, and GI are no longer equal to $\mathrm{CP}^{\prime}, \mathrm{WI}^{\prime}$, and $\mathrm{GI}^{\prime}$, respectively.

Since the last $L_{\text {WI }}$ samples are affected by the window, we define a new region for them which we call GI2' . Thus, now 


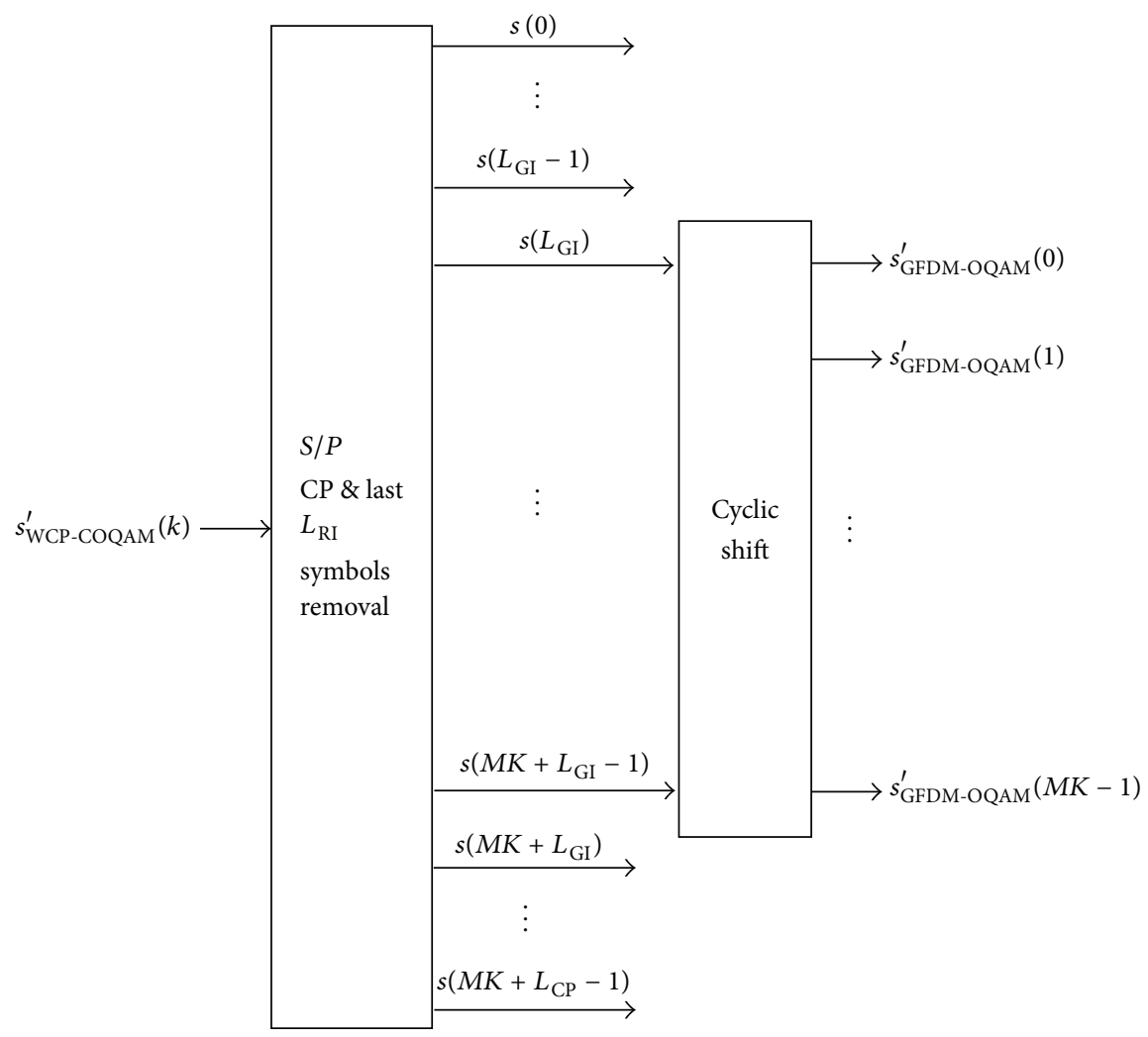

FIGURE 7: Block diagram of one GFDM-OQAM block recovery process from a WCP-COQAM block.

the original GI and $\mathrm{GI}^{\prime}$ regions are divided into GI1 and GI2 and $\mathrm{GI}^{\prime}$ and $\mathrm{GI} 2^{\prime}$, respectively, so that $\mathrm{WI} \neq \mathrm{WI}^{\prime}, \mathrm{GI} 1=$ $\mathrm{GI}^{\prime}$, and $\mathrm{GI} 2 \neq \mathrm{GI} 2^{\prime}$. As for the lengths of these regions, $L_{\mathrm{WI}}=L_{\mathrm{WI}^{\prime}}=L_{\mathrm{GI} 2}=L_{\mathrm{GI} 2^{\prime}}$ and $L_{\mathrm{GI} 1}=L_{\mathrm{GI} 1^{\prime}}$. Note that GI1 and $\mathrm{GI} 1^{\prime}$ are now the only really redundant parts. Because of that, only GI1 and not the whole GI (as it is explained in [21]) acts as a real guard interval against multipath channels when frequency domain equalization (FDE) is performed. Hence, $L_{\mathrm{GI} 1} \geq L_{\mathrm{CH}}-1$ must be fulfilled in order to maintain full orthogonality through a multipath channel, where $L_{\mathrm{CH}}$ is the transmission channel length. Thus, considering that $L_{\mathrm{GI} 2}=$ $L_{\mathrm{WI}}$ and so $L_{\mathrm{GI} 1}=L_{\mathrm{GI}}-L_{\mathrm{GI} 2}=L_{\mathrm{GI}}-L_{\mathrm{WI}}$, we can define the full orthogonality condition for WCP-COQAM as

$$
L_{\mathrm{GI}} \geq L_{\mathrm{CH}}-1+L_{\mathrm{WI}} .
$$

It is worth mentioning that the FDE should be performed for samples between GI2 and GI1', inclusive, because that is the section corresponding to the cyclic convolution between GI1 and GI1' with the transmission channel.

3.2. Spectral Efficiency Analysis. Figure 9 shows how overlapping between adjacent blocks is performed in WCPCOQAM. Every windowed region is overlapped with the next or the previous block, so that the WI region of each block is overlapped with the GI2' region of the previous block. Thus, the samples within WI regions do not suppose an overload and they do not affect the SE. Based on this fact, in [21], the authors claim that the use of the window function has no effect on SE. However, this statement is not precise. Although the samples in WI do not directly affect SE, because they overlap with the samples of GI2' of the previous block, windowing implies adding the GI2 region in order to fulfil the orthogonality condition defined in (12). Note that the use of the window function and overlapping of WI and GI2' regions distort those samples in GI2' region. This and the fact that FDE is performed between GI2 and GI1', inclusive, imply that the GI2 region is necessary in order to recover those samples originally placed in the GI2' region. At this point, we conclude that both GI1 and GI2 are essential parts within the GI region of WCP-COQAM.

In order to make a fair analysis of SE in WCP-COQAM, we compare it to GFDM-OQAM, because both modulation schemes only differ in windowing and overlapping processes. In order to maintain a homogeneous notation between GFDM-OQAM and WCP-COQAM, we assume the following considerations: (for the sake of readability, from this point on, parameters corresponding to OFDM will be expressed with the subindex $O$, parameters corresponding to GFDM-OQAM will be expressed with the subindex $G$, and parameters corresponding to WCP-COQAM will be expressed with the subindex $W$ ).

$$
\begin{aligned}
\mathrm{CP}_{G} & =\mathrm{GI}_{G}=\mathrm{GI}_{G}, \\
\mathrm{CP}_{W} & =\left[\mathrm{WI}_{W}, \mathrm{GI}_{W}\right], \\
\mathrm{GI}_{W} & =\left[\mathrm{GI}_{W}, \mathrm{GI} 2_{W}\right] .
\end{aligned}
$$


Block $l$

\begin{tabular}{|c|c|c|c|c|c|c|}
\multicolumn{9}{|c|}{ (GFDM-OQAM block $l$} \\
\hline \multicolumn{2}{|c|}{} \\
\hline
\end{tabular}

(GFDM-OQAM block without CP extension)

(a)

\begin{tabular}{|l|l|l|l|l|l|l|l|}
\hline \multicolumn{2}{|c|}{ Block $l$} \\
\hline \multicolumn{1}{|c|}{} \\
\hline
\end{tabular}

(b)

FIGURE 8: Windowing operation of a WCP-COQAM block. (a) A WCP-COQAM block before windowing is equal to a GFDM-OQAM block with CP extension. (b) The first and the last $L_{\mathrm{WI}}$ samples of a WCP-COQAM block are distorted by the window.

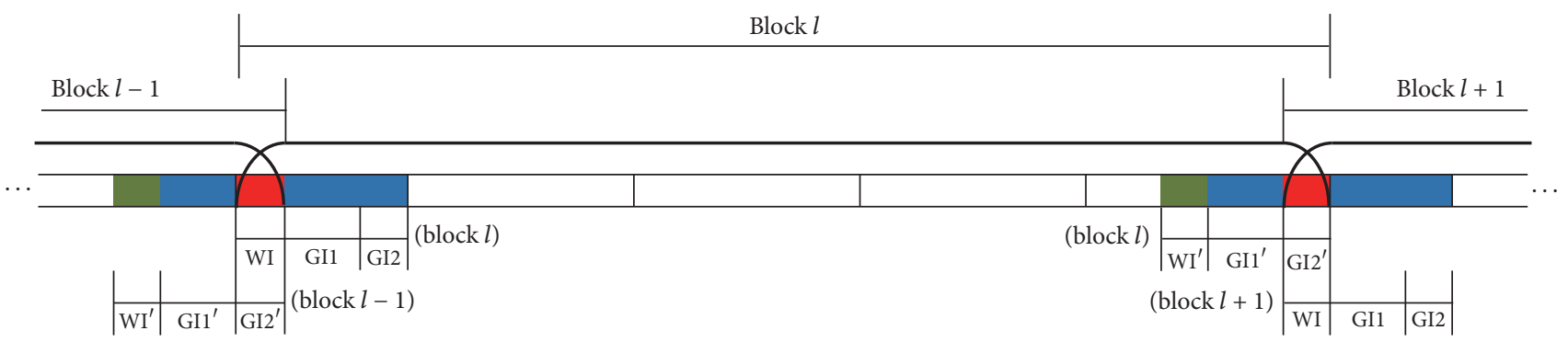

FIGURE 9: Overlapping between adjacent WCP-COQAM blocks and CP extension's structure. Only GI1 and GI1' remain equal in order to emulate a circular convolution with the channel and perform frequency domain equalization, so GI2 implies an overload that affects SE.

The criterion we applied for this comparison is to provide equal protection against multipath channels for both modulations, so that $L_{\mathrm{GI}_{G}}=L_{\mathrm{GI1}_{W}}$. Thus

$$
\begin{aligned}
\mathrm{SE}_{G} & =\frac{M K}{L_{\mathrm{GI}_{G}}+M K}=\frac{M K}{L_{\mathrm{GI}_{G}}+M K}, \\
\mathrm{SE}_{W} & =\frac{M K}{L_{\mathrm{GI}_{W}}+M K}=\frac{M K}{L_{\mathrm{GI}_{W}}+L_{\mathrm{GI}_{W}}+M K} .
\end{aligned}
$$

From this analysis, it is clear that if full orthogonality is to be maintained, the windowing process brings an extra GI2 region to the $\mathrm{CP}$ which has effects on the SE of a WCPCOQAM signal. In particular, SE is reduced by a factor of $\left(L_{\mathrm{GI} 1}+L_{\mathrm{GI} 2}+M K\right) /\left(L_{\mathrm{GI}}+M K\right)$ compared to a windowless system with equal multipath channel protection.

\section{Simulation Results and Discussion}

In this section, we show and discuss the results obtained from the simulations of the systems previously described. Based on these results, we assess the performances of the simulated systems in terms of BER, PSD, and SE. In our analysis, we prioritize robustness against highly dispersive channels, so that we use the BER of transmissions through Rayleigh fading multipath channels in order to assess the robustness of the MCM systems. In addition to BER, we also take into consideration the PSD of the transmitted signals, for it is an important factor when it comes to CR systems. Regarding SE, we use it to represent the cost of the CP extensions, windowing process, and convolution tails caused by the prototype filters in FBMC-OQAM, so that we can obtain a fair comparison between different MCM systems. 
TABLE 1: Basic simulation parameters. Unless opposite is stated, the results shown in this work have been obtained under the conditions defined by these parameters.

\begin{tabular}{|c|c|c|c|c|}
\hline & OFDM & FBMC-OQAM & GFDM-OQAM & WCP-COQAM \\
\hline Signal bandwidth & & & $20 \mathrm{MHz}$ & \\
\hline IQ modulation & & & QPSK & \\
\hline$M$ (subcarriers) & & & 512 & \\
\hline$K$ (symbols/block; overlapping factor) & 1 & 4 & 4 & 4 \\
\hline$L_{\mathrm{CP}}$ (samples) & 32 & - & 32 & 224 \\
\hline$L_{\mathrm{WI}}$ (samples) & - & - & - & 96 \\
\hline$L_{\mathrm{GI}}$ (samples) & 32 & - & 32 & 128 \\
\hline$L_{\mathrm{GI} 1}$ (samples) & - & - & - & 32 \\
\hline$L_{\mathrm{GI} 2}$ (samples) & - & - & - & 96 \\
\hline Prototype filter & - & & PHYDYAS [5] & \\
\hline$L_{p}$ (samples) & - & & 2048 & \\
\hline
\end{tabular}

Since we are evaluating MCM systems, we consider it appropriate to compare the analysed FMC modulation techniques with an OFDM reference system in order to assess their performance. Table 1 shows the configuration parameters we used in most of our simulations. We choose parameters suitable for low-band transmissions in ultrareliable and low-latency indoor scenarios. For that, we consider aspects like subcarrier bandwidth and transmission channel's coherence bandwidth, data block duration, and window length for PSD improvement.

4.1. Robustness against Multipath Channels. In this subsection, we analyse the robustness of each MCM system against multipath channels. In order to make a fair comparison, we simulate every system with equal protection against multipath channels, matching effective GI lengths $\left(L_{\mathrm{GI}_{\mathrm{O}}}\right.$, $L_{\mathrm{GI}_{G}}$, and $\left.L_{\mathrm{GII}_{W}}\right)$. These parameters are selected as shown in Table 1.

The equalization technique we use in our simulations is one-tap zero-forcing (ZF) FDE for all the MCM systems. While OFDM, GFDM-OQAM, and WCP-COQAM employ $\mathrm{CP}$ extensions to prevent ISI caused by the transmission channel, FBMC-OQAM could be considered a special case in this aspect. Since it is not a blockwise modulation and no $\mathrm{CP}$ extension is used, one-tap FDE is not a straightforward equalization technique for FBMC-OQAM. However, due to the subcarrier bandwidth and symbol duration near to transmission channel's coherence bandwidth and time, respectively, we consider the one-tap FDE as a suitable equalization method for FBMC-OQAM in this case. An indepth analysis about the doubly dispersive channels' impact on FBMC systems is given in [28].

We simulated transmissions through a Rayleigh fading channel model with exponential power delay profile (PDP) whose root mean square (rms) delay spread and channel length are $t_{\mathrm{rms}}=185 \mathrm{~ns}$ and $L_{\mathrm{CH}}=22$ taps. Besides, we simulate uncoded and coded communications, with turbo codes, soft decoding, and a code rate of 1/3. In Figure 10,

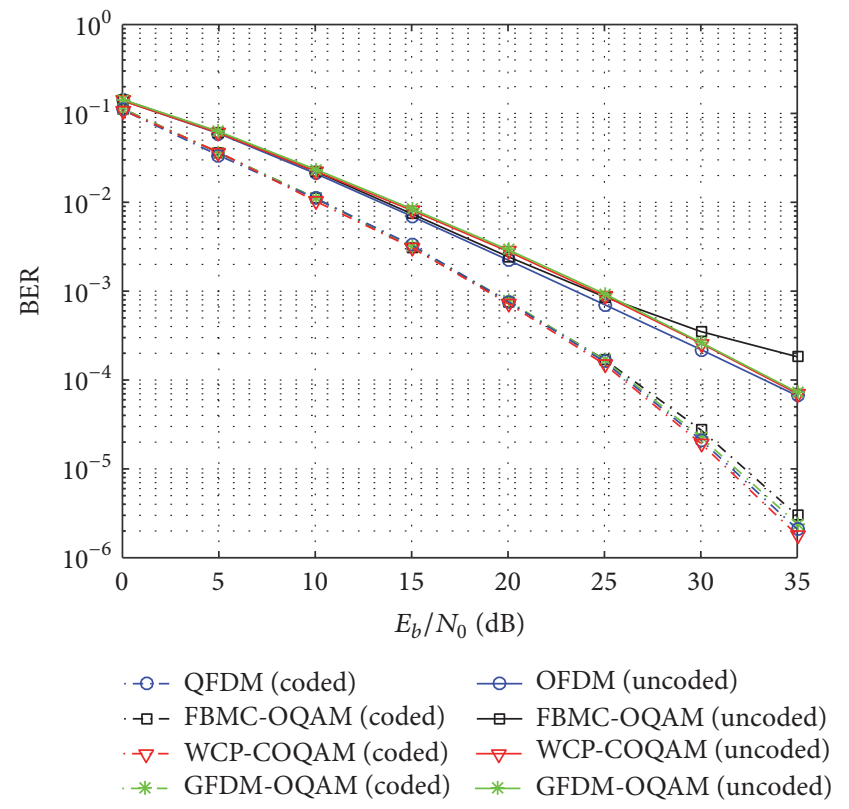

FIGURE 10: BER curves of the analysed MCM systems in a multipath channel. We employed turbo encoding/decoding with soft decoding and a code rate of $1 / 3$.

we compare the BER versus $E_{b} / N_{0}$ of the MCM systems over these channel models, assuming perfect synchronization and full channel state information (CSI). We got these BER curves by simulating transmissions through $10^{4}$ Monte Carlo iterations for each MCM system and each channel model. We transmit 12288 random data bits and we generate new random channels at each Monte Carlo iteration.

Here we show that, for uncoded transmissions, in terms of error rate, FBMC-OQAM performs slightly worse than the rest of MCM systems under the simulated conditions. While at low $E_{b} / N_{0}$ values every MCM system provides similar BER, an error floor appears at high $E_{b} / N_{0}$ values because of the lack of GI in FBMC-OQAM. On the other hand, although 


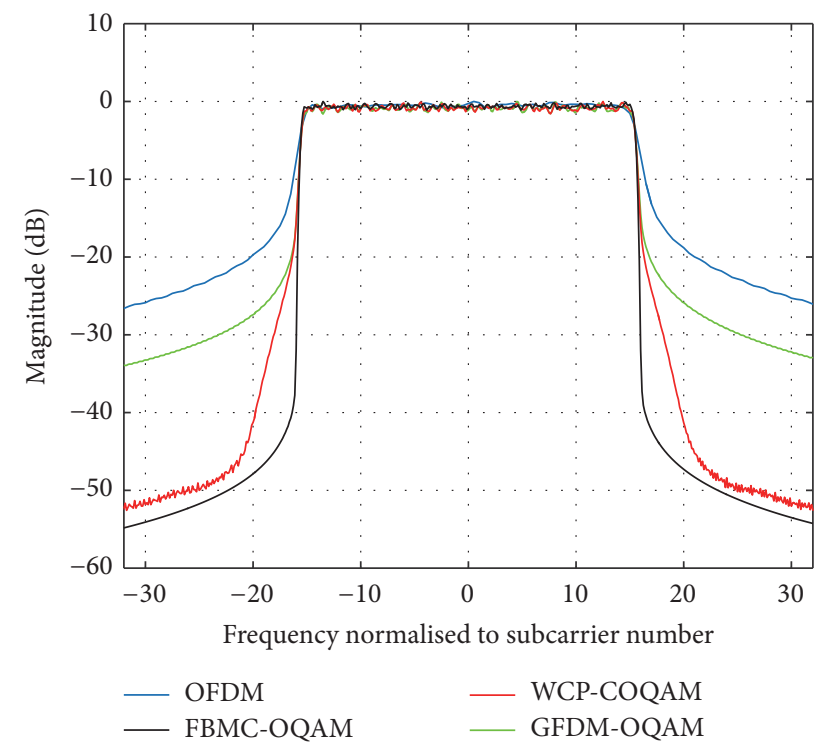

FIGURE 11: PSD of the analysed MCM systems with some subcarriers deactivated. Only the central 32 subcarriers out of 512 are activated.

OFDM, GFDM-OQAM, and WCP-COQAM provide similar performances in terms of BER providing equal multipath effect protection, OFDM slightly outperforms the other two MCM schemes due to its perfect orthogonality.

Finally, it is worth noting that, for coded transmissions, all the simulated MCM systems present similar BER curves, so that they provide similar robustness against multipath channels.

4.2. Power Spectral Density Analysis. PSD is a relevant characteristic in order to design a CR communication system suitable for scenarios with high spectrum occupation. In this subsection, we compare the spectrums of the signals of the analysed MCM systems in order to assess their potential suitability for CR based systems.

Figure 11 shows the spectrums of OFDM, GFDMOQAM, WCP-COQAM, and FBMC-OQAM signals one on top of the other. For this test, we only activate the 32 central subcarriers in order to make the power leakage into adjacent subcarriers visible.

As shown in Figure 11, the FB based MCM systems carry significant reduction of OOB radiation compared to OFDM. However, even between these MCM schemes, there is still a remarkable difference. In this sense, FBMC-OQAM outperforms both GFDM-OQAM and WCP-COQAM. On the other hand, thanks to the windowing, WCP-COQAM shows considerably lower OOB radiation than GFDM-OQAM. It is worth mentioning that the longer the WI region is, the narrower WCP-COQAM signal's spectrum gets. Since extra samples within the GI region are needed for windowing, as described in Section 3, PSD improvement of WCP-COQAM over GFDM-OQAM comes at the cost of lower SE. More detailed analysis on SE is given in Section 4.3.
4.3. Spectral Efficiency Analysis. In this subsection, we analyse SE in order to evaluate the cost of obtaining higher robustness against dispersive channels and better PSD by means of CP extensions and windowing. For this analysis, we consider the parameters defined in Table 1, so that effective multipath channel protection is equal for OFDM, GFDMOQAM, and WCP-COQAM. Equations (15) show the SE values of the three blockwise MCM systems:

$$
\begin{aligned}
& \mathrm{SE}_{\mathrm{O}}=\frac{M K_{O}}{L_{\mathrm{GI}_{O}}+M K_{O}}=0.9412, \\
& \mathrm{SE}_{G}=\frac{M K_{G}}{L_{\mathrm{GI}_{G}}+M K_{G}}=0.9846, \\
& \mathrm{SE}_{W}=\frac{M K_{W}}{L_{\mathrm{GI}_{W}}+M K_{W}}=0.9412 .
\end{aligned}
$$

Here we show a particular case with some specific parameters. On one hand, GFDM-OQAM presents higher SE than OFDM because its GI precedes $K$ symbols, while in OFDM the GI precedes only one symbol. On the other hand, because of the windowing operation, the $\mathrm{CP}$ extension of WCPCOQAM, specifically the GI part, has $L_{\mathrm{GI} 2}$ extra samples with respect to GFDM-OQAM and that is the reason why WCP-COQAM also presents lower SE than GFDM-OQAM. However, under these conditions, with a high number of subcarriers, we can conclude that the differences in the SE between OFDM, GFDM-OQAM, and WCP-COQAM are not really significant.

Regarding FBMC-OQAM, once again, we consider it different from the rest of MCM systems because it is not a blockwise modulation and it uses no GI protection for multipath effect. So its SE depends on the length of the transmitted frames with respect to the convolution tails. For long data transmissions, its SE will tend to 1, while for short data transmissions it should tend to 0.5 , assuming a minimum amount of data samples equivalent to a GFDMOQAM or WCP-COQAM block. This calculation is simple if we introduce 4 complex data symbols (8 OQAM symbols) in (2).

$$
a_{m}(n)=\sum_{k=0}^{7} x_{m}(k) \delta\left(n-\frac{k M}{2}\right), \quad n \in\left[0, \frac{8 M}{2}-1\right]
$$

and we introduce these upsampled data in (3). The resultant $y(n)$ signal will contain 4095 samples, of which half will correspond to actual data and the rest to convolution tails from subcarrier filtering.

Considering the low-latency scenarios our research focuses on, SE might suppose a significant drawback for FBMC-OQAM compared to GFDM-OQAM and WCPCOQAM during short data transmissions.

\section{Conclusions}

In this article, we make a comparison between three candidates for PHY layer modulation in 5G and a reference OFDM 
system in order to assess their suitability for industrial wireless communications based on CR. For that, we simulate lowband communications and highly dispersive indoor channel scenarios. Under these conditions, we show the performances and features of these MCM systems from different points of view.

Regarding robustness against multipath channels, we conclude that all the analysed MCM systems provide similar performances under the simulated conditions. Although for uncoded transmissions FBMC-OQAM presents an error floor at high $E_{b} / N_{0}$ values, this error floor is corrected when coding is used.

In terms of PSD and OOB radiation, these filtered MCM schemes provide more restrained spectrum than OFDM. In particular, FBMC-OQAM outperforms the rest of the modulation schemes in this aspect, so it could be a suitable candidate for the $\mathrm{CR}$ scenarios considered in our research. On the other hand, although not as good as FBMC-OQAM, WCPCOQAM still has considerably better PSD than GFDMOQAM, which only provides less than $10 \mathrm{~dB}$ of difference in OOB radiation with respect to OFDM. Therefore, we conclude that circular filtering only is not sufficient and windowing must be applied in order to ensure more efficient use of the spectrum. Moreover, we consider that, due to its also restrained spectrum, WCP-COQAM might be another suitable modulation scheme for CR applications.

As for the SE analysis, as discussed in Section 4.3, the difference between OFDM, GFDM-OQAM, and WCPCOQAM is not really significant for the parameters we used in our simulations. On the other hand, FBMC-OQAM might suffer from severe degradation of SE during short data transmissions in the low-latency scenarios we consider in our research.

\section{Future Work}

In this work, we considered perfect synchronization and full channel state information at the receiver, but it is also important to consider more realistic simulation conditions in the future. So the next step in our investigation will be to analyse and propose time-frequency synchronization and channel estimation methods for GFDM-OQAM and WCPCOQAM.

The other key point in our research will be to simulate channel models that fairly emulate industrial wireless channel conditions. For that, we will simulate severe multipath conditions, time-varying channels, impulsive noise, and interference from other wireless systems.

\section{Conflicts of Interest}

The authors declare that there are no conflicts of interest regarding the publication of this paper.

\section{Acknowledgments}

This work was partly supported by TIPOTRANS (ETORTEK) and CIIRCOS (PC2013-68) projects of the Basque Government (Spain) and by COWITRACC
(TEC2014-59490-C2-1-P/2-P) project of the Spanish Ministry of Economy and Competitiveness.

\section{References}

[1] H. Zhang, D. L. Ruyet, M. Terré et al., "Application of the FBMC physical layer in a Cognitive Radio scenario," Tech. Rep., PHYDYAS-Physical Layer for Dynamic Access and Cognitive Radio, 2009.

[2] I. Gaspar and G. Wunder, "5G cellular communications scenarios and system requirements," Tech. Rep., 5th Generation NonOrthogonal Waveforms for Asynchronous Signalling, 2013.

[3] P. Popovski, V. Braun, H. Mayer et al., "Scenarios, requirements and KPIs for 5G mobile and wireless system," Tech. Rep., Mobile and wireless communications Enablers for the Twenty-twenty Information Society (METIS), 2013.

[4] X. Mestre, D. Gregoratti, M. Renfors et al., "ICT-EMPhAtiC final project report," Tech. Rep., Enhanced Multicarrier Techniques for Professional Ad-Hoc and Cell-Based Communications, 2015.

[5] A. Viholainen, M. Bellanger, and M. Huchard, "Prototype filter and structure optimization," PHYDYAS-Physical Layer for Dynamic Access and Cognitive Radio, 2009.

[6] N. Michailow, M. Matthe, I. S. Gaspar et al., "Generalized frequency division multiplexing for 5 th generation cellular networks," IEEE Transactions on Communications, vol. 62, no. 9, pp. 3045-3061, 2014.

[7] V. Vakilian, T. Wild, F. Schaich, S. Ten Brink, and J.-F. Frigon, "Universal-filtered multi-carrier technique for wireless systems beyond LTE," in Proceedings of the 2013 IEEE Globecom Workshops, GC Wkshps 2013, pp. 223-228, IEEE, December 2013.

[8] M. Agiwal, A. Roy, and N. Saxena, "Next generation 5G wireless networks: A comprehensive survey," IEEE Communications Surveys and Tutorials, vol. 18, no. 3, pp. 1617-1655, 2016.

[9] X. Zhang, M. Jia, L. Chen, J. Ma, and J. Qiu, "Filtered-OFDMenabler for flexible waveform in the 5th generation cellular networks," in Proceedings of the 58th IEEE Global Communications Conference (GLOBECOM '15), pp. 1-6, IEEE, December 2015.

[10] L. Zhang, A. Ijaz, P. Xiao, A. Quddus, and R. Tafazolli, "Subband filtered multi-carrier systems for multi-service wireless communications," IEEE Transactions on Wireless Communications, vol. 16, no. 3, pp. 1893-1907, 2017.

[11] A. Lizeaga, M. Mendicute, P. M. Rodriguez, and I. Val, "Evaluation of WCP-COQAM, GFDM-OQAM and FBMC-OQAM for industrial wireless communications with Cognitive Radio," in Proceedings of the 2017 IEEE International Workshop of Electronics, Control, Measurement, Signals and their Application to Mechatronics (ECMSM), pp. 1-6, Donostia, Spain, May 2017.

[12] P. M. Rodriguez, I. Val, A. Lizeaga, and M. Mendicute, "Evaluation of cognitive radio for mission-critical and timecritical WSAN in industrial environments under interference," in Proceedings of the 2015 11th IEEE World Conference on Factory Communication Systems (WFCS '15), pp. 1-4, May 2015.

[13] P. M. Rodríguez, R. Torrego, F. Casado et al., "Dynamic spectrum access integrated in a wideband cognitive RF-ethernet bridge for industrial control applications," Journal of Signal Processing Systems, vol. 83, no. 1, pp. 19-28, 2016.

[14] A. K. Somappa, K. Ovsthus, and L. Kristensen, "An industrial perspective on wireless sensor networks - a survey of requirements, protocols, and challenges," IEEE Communications Surveys Tutorials, vol. 16, pp. 1391-1412, 2014. 
[15] S. Vitturi, "Wireless networks for the factory floor: requirements, available technologies, research trends and practical applications," in Proceedings of the IEEE International Conference on Emerging Technologies and Factory Automation, 2014.

[16] P. Popovski, "Ultra-reliable communication in 5G wireless systems," in Proceedings of the 2014 1st International Conference on $5 G$ for Ubiquitous Connectivity (5GU '14), pp. 146-151, November 2014.

[17] N. Brahmi, O. N. C. Yilmaz, K. W. Helmersson, S. A. Ashraf, and J. Torsner, "Deployment strategies for ultra-reliable and lowlatency communication in factory automation," in Proceedings of the IEEE Globecom Workshops (GC Wkshps '15), pp. 1-6, IEEE, December 2015.

[18] O. N. Yilmaz, "Ultra-Reliable and Low-Latency 5G Communication," in Proceedings of the European Conference on Networks and Communications (EuCNC '16), 2016.

[19] H. Lin and P. Siohan, "FBMC/COQAM: an enabler for cognitive radio," in Proceedings of the 4th International Conference on Advances in Cognitive Radio (COCORA '14), pp. 8097-8101, May 2014.

[20] H. Lin and P. Siohan, "An advanced multi-carrier modulation for future radio systems," in Proceedings of the 2014 IEEE International Conference on Acoustics, Speech, and Signal Processing (ICASSP '14), pp. 8097-8101, May 2014.

[21] H. Lin and P. Siohan, "Multi-carrier modulation analysis and WCP-COQAM proposal," Eurasip Journal on Advances in Signal Processing, vol. 2014, no. 1, article 79, 2014.

[22] F. Luo and C. J. Zhang, Signal Processing for 5G: Algorithms and Implementations, chapter 8, John Wiley \& Sons, Ltd, Chichester, UK, 2016.

[23] J. J. Benedetto, C. Heil, and D. F. Walnut, Gabor Systems and the Balian-Low Theorem, Birkhäuser, chapter 2, Boston, Mass, USA, 1998.

[24] A. Ijaz, L. Zhang, P. Xiao, and R. Tafazolli, Analysis of Candidate Waveforms for 5 G Cellular Systems, chapter 1, InTech, 2016.

[25] R. Gerzaguet, N. Bartzoudis, L. G. Baltar et al., "The 5G candidate waveform race: a comparison of complexity and performance," Eurasip Journal on Wireless Communications and Networking, vol. 2017, no. 1, article 13, 2017.

[26] A. B. Üçüncü and A. Ö. Yilmaz, "Pulse shaping methods for OQAM/OFDM and WCP-COQAM," https://arxiv.org/ abs/1509.00977.

[27] F. Luo and C. J. Zhang, Signal Processing for 5G: Algorithms and Implementations, John Wiley \& Sons, Ltd, Chichester, UK, 2016.

[28] L. Zhang, P. Xiao, A. Zafar, A. ul Quddus, and R. Tafazolli, "FBMC system: an insight into doubly dispersive channel impact," IEEE Transactions on Vehicular Technology, vol. 66, no. 5, pp. 3942-3956, 2017. 


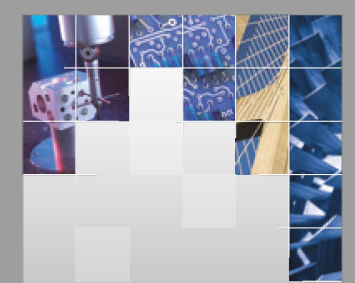

\section{Enfincering}
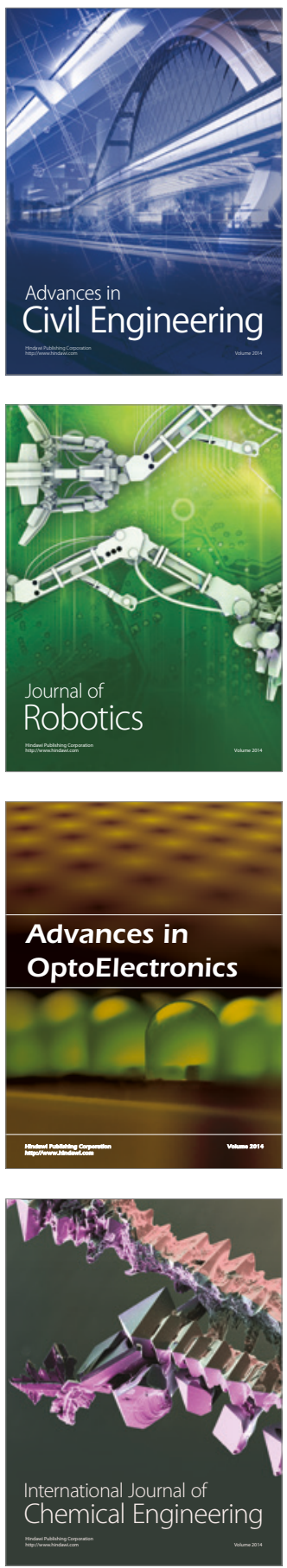

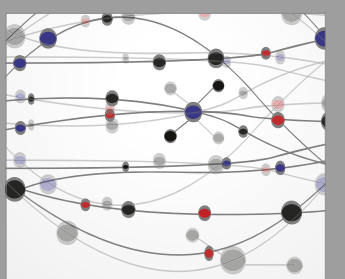

The Scientific World Journal

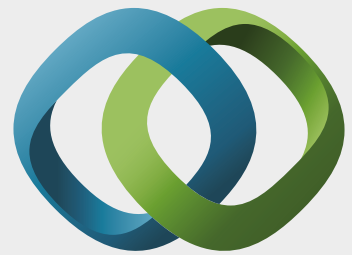

\section{Hindawi}

Submit your manuscripts at

https://www.hindawi.com
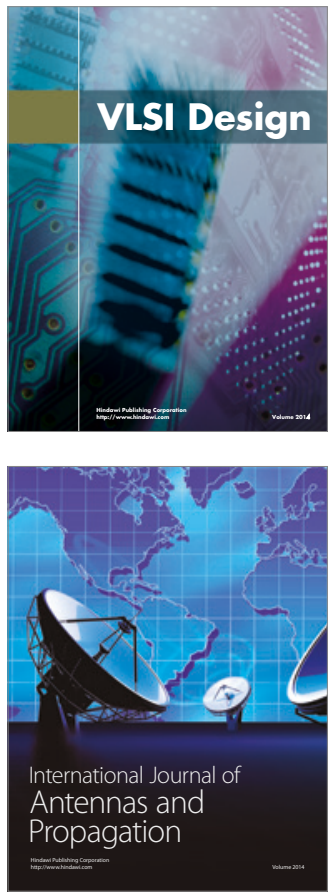

\section{Rotating}

Machinery
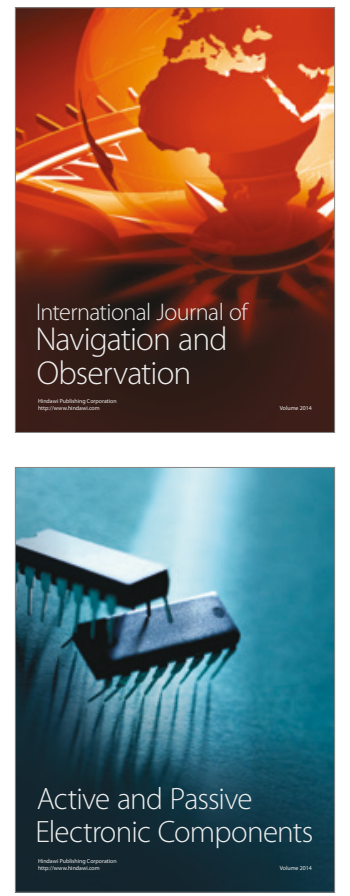
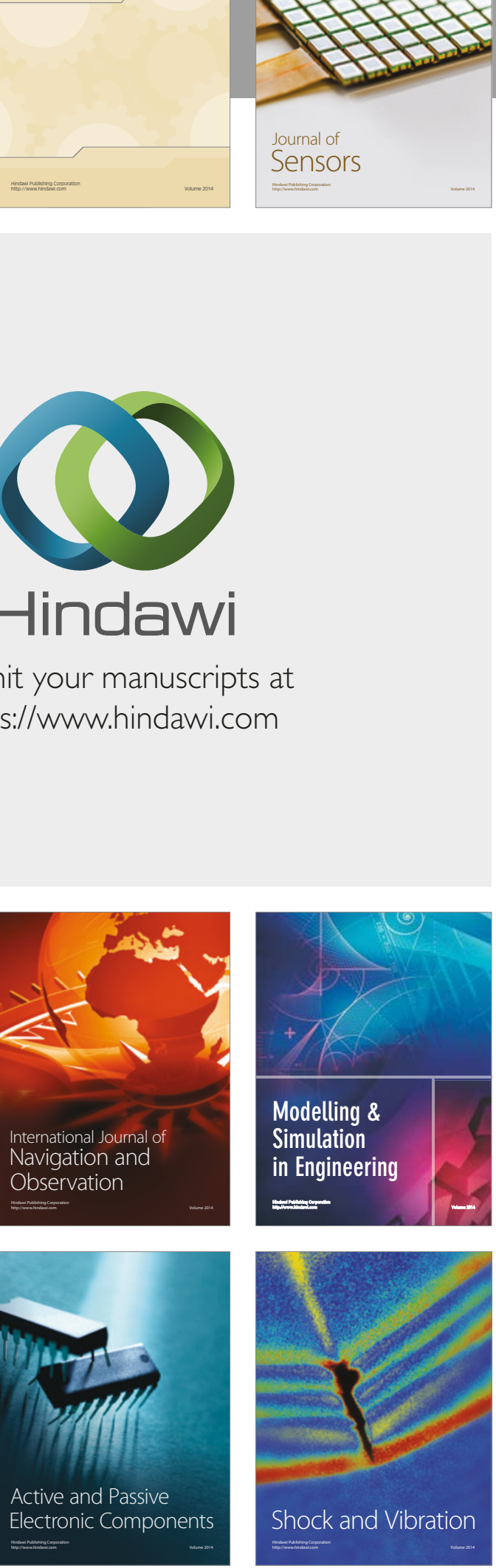
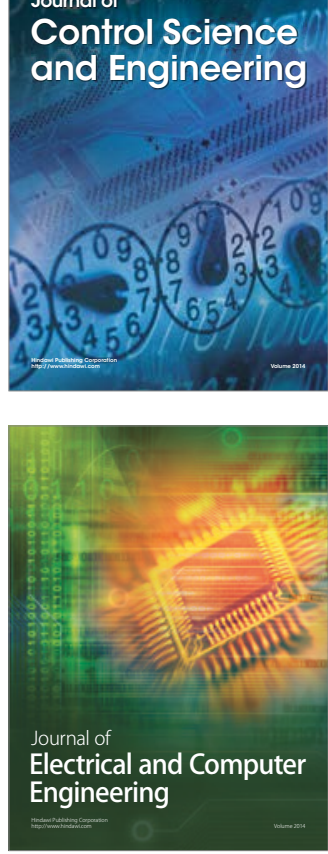

Distributed

Journal of

Control Science

and Engineering
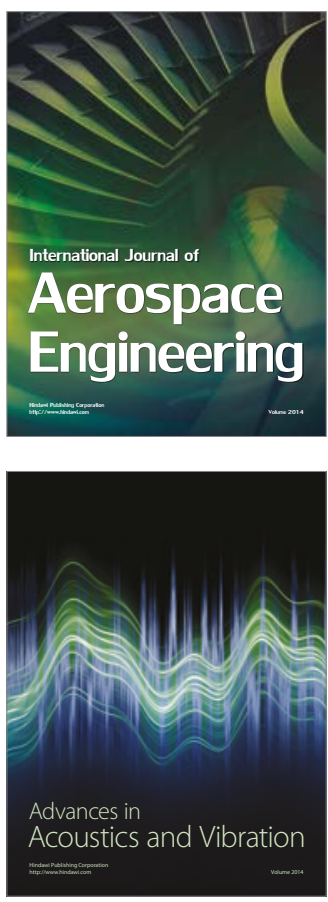

Sensor Networks 